\title{
Do women and men respond differently to alcohol use disorder treatment?
}

\author{
Newberry Sydne ${ }^{1}$, Gore Kristie ${ }^{1}$, Belsher Bradley ${ }^{2}$, Chloe Bird ${ }^{1}$ and Hempel Susanne ${ }^{1,3 *}$ \\ ${ }^{1}$ RAND Corporation, USA \\ ${ }^{2}$ Defense Health Agency, Psychological Health Centre of Excellence, USA \\ ${ }^{3}$ Southern California Evidence Review Centre, University of Southern California (USC), USA
}

\section{Introduction}

Alcohol use is a leading cause of disease and death worldwide. As of 2015 , the lifetime prevalence for alcohol use disorder for U.S. adults was 29 percent [1]. The annual rates are considerably higher for men than for women ( 8.4 vs. 4.2 percent). In the U.S. Military, rates of alcohol use disorders are wide-ranging depending on the setting in which they are assessed [2]. In a 2015 survey, active-duty men were substantially more likely than their female counterparts to report binge drinking (31.2 percent compared with 23.0 percent) or heavy drinking (6.0 percent compared with 1.3 percent). However, men and women were equally likely to report suffering serious and work-related consequences from drinking [3].

Clinical practice guidelines for the management of substance use disorders such as the guideline by the Department of Veterans Affairs (VA)/Department of Defense (DoD) [4], recommend evidencedbased pharmacologic (the opiate antagonist naltrexone, disulfiram, topiramate, and acamprosate) and psychosocial (cognitive behavioural therapy, motivational enhancement therapy, community reinforcement approach, and 12-step facilitation) approaches to the management of alcohol use disorder. Yet whether women and men respond differently to recommended treatments for alcohol use disorder, given the evidence of differences in alcohol use, is unknown.

The Defense Health Agency Psychological Health Center of Excellence (PHCoE) commissioned the RAND Corporation to conduct a systematic review to determine whether women respond differently than men to guideline recommended treatments for alcohol use disorder. The rates of alcohol use disorder among service personnel, particularly female service members, and the most-effective treatments are of concern to military psychological health practitioners. Findings would be of interest to military and civilian health policymakers and practitioners who oversee or implement and seek to understand factors that might contribute to differences in the response to treatment for alcohol use disorder.

In order to address the question of gender differences objectively, we developed an innovative method to identify research studies and to analyse gender differences in responses to alcohol use disorder treatment. The project set out to determine whether gender differences in treatment response exist, and information about both the presence and the absence of gender differences, were considered important outcomes of the evidence review. In this commentary we summarize our methods and findings and highlight the need for further research that puts more emphasis on women's health [5].

\section{Methods to identify gender differences and results}

For our comprehensive evidence report we searched both research databases (PubMed, PsycINFO, Embase, Cochrane Central Register of Controlled Trials, Cochrane Database of Systematic Reviews, Clinicaltrials.gov) and bibliographies of existing systematic reviews for randomized controlled trials (RCTs) evaluating evidence-based treatment of alcohol use disorder. In addition, we contacted authors of trials in U.S. patients, published between 1997 and March 2018, to obtain information on the presence or the absence of gender differences in treatment responses. Importantly, we did not directly search for gender differences so as to not bias the sample towards studies emphasizing differences between men and women. Instead, we obtained all reports of RCTs evaluating alcohol use disorder treatment and reviewed the full text of the articles for any information on gender differences or analyses stratified by gender.

To be included in the systematic review, publications had to report on an analysis of gender differences in the effects of pharmacologic and/ or psychosocial treatments for adults who met diagnostic criteria for alcohol use disorder. Two reviewers independently screened literature identified by the searches using predetermined eligibility criteria, abstracted data, and critically appraised publications. We included studies that reported data stratified by gender, documented gender effects on treatment responses, or included only men or only women. We were equally interested in the presence as well as the absence of empirical evidence of gender differences and screened studies for the assessment of effects of gender or data presented separately for women and for men.

We summarized gender differences in the treatment responses as differences between genders in the difference between the intervention and control group results (difference of differences [DiffoDiff]) together with the 95-percent confidence interval (CI). Meta-analyses were conducted to determine the DiffoDiff for effects in studies that directly compared outcomes for men and women. Meta-regressions were conducted to assess gender differences indirectly across studies that enrolled only men, only women, or both (but reported results separately for men and women). The quality of evidence was assessed

*Correspondence to: Susanne Hempel, Southern California Evidence Review Centre, University of Southern California, Los Angeles, USA, Tel: +1 310482 8209; E-mail: susanneh@usc.edu

Received: September 02, 2019; Accepted: September 16, 2019; Published: September 20, 2019 
using the Grades of Recommendation, Assessment, Development, and Evaluation (GRADE) approach.

We screened 13,771 citations and reviewed the full text of 1,434 publications. We identified 23 original studies. For one additional study of Army personnel that did not report findings by gender, the investigators shared their data, bringing the total to 24 included primary research studies.

While some individual studies found evidence of gender differences, we did not find systematic gender differences in treatment effects across studies. The analysis of seven RCTs that included women and men and reported on a treatment response measure did not detect systematic gender differences (DiffoDiff 0.42; CI -0.80, 1.64). Similarly, indirect comparisons across studies in women or men and studies including both genders showed no statistically significant effect (DiffoDiff 0.03; CI $-0.28,0.34 ; \mathrm{p}=0.840$ ). We found conflicting results in individual studies for treatment compliance, and no statistically significant effect was detected in indirect comparisons across 11 RCTs contributing to the analysis (DiffoDiff 0.31 ; CI $-0.03,0.65 ; \mathrm{p}=0.073$ ). Only two studies reported on adverse events and both evaluated naltrexone: The studies reported more findings of nausea in women taking naltrexone than in men (rates of nausea were not related to rates of heavy drinking). Stratifying interventions by pharmacological, psychological, or combination treatment indicated larger effects for women in combination treatment, but the result was based on only one study without replication and should to be interpreted with caution.

We found no indication that gender differences in symptom improvement were affected by duration $(\mathrm{p}=0.721)$ or intensity $(\mathrm{p}=0.270)$ of treatment, but the small number of studies contributing to the analyses limits the interpretation of the finding. We could not determine whether gender differences vary by treatment setting (only one study was conducted in primary care).

\section{Discussion and implications}

After screening nearly 14,000 citations, only 24 studies met our inclusion criteria. Thus, the most important finding of our work is the need for researchers to assess gender differences in alcohol use disorder treatment effectiveness and safety.

Although some of our analyses identified trends that favoured men or women for certain treatments, we saw no systematic differences for outcomes across groups of studies of the same treatments. Only two studies compared rates and kinds of adverse effects of treatment for men and women; both were studies of a pharmacologic agent, and the findings were inconsistent. Furthermore, individual treatment effectiveness is nearly always affected by compliance, yet we found no systematic differences in treatment compliance between men and women, either for pharmacological treatments or psychosocial treatments; and superior effectiveness of treatment for one gender or the other in individual studies was never attributed to better compliance.

The small number of studies precluded an assessment of further questions of interest, including differences between men and women in the comparative effectiveness of pharmacologic agents, between pharmacologic agents and psychosocial therapy, or between pharmacologic agents accompanied by psychosocial therapy and the same agents without psychosocial therapy. Moreover, we could not assess whether differences in study duration (either treatment duration or follow-up time), treatment intensity (for example, drug dose or frequency of therapy sessions), or treatment setting affected treatment effectiveness in men differently than in women.

Biopsychosocial differences between men and women may be associated with differential rates of alcohol use disorder and treatment response patterns. For example, hormonal and metabolic differences between men and women may influence the effects of pharmacological treatments. Differential rates of comorbid mental health conditions, such as depression and posttraumatic stress disorder may be another reason men and women with alcohol use disorder could respond differently to treatment. However, despite the research volume on alcohol use disorder and the large number of intervention evaluations, we cannot answer the question of whether men and women respond eqully to treatment approaches.

Our review showed a profound lack of information on the presence and absence of gender differences. In addition, studies that met inclusion criteria tended to be smaller and enrolled only few women. The studies also tended to assess a wide variety of outcomes, making cross-study comparisons difficult. The number of available assessment tools used in alcohol use disorder research presents a challenge to the field. For example, studies reported numerous different outcomes (e.g., hazardous drinking, binge drinking, frequency of drinking) to measure treatment response which makes it challenging to compare results across studies. Reporting common data elements is an important way to facilitate comparisons across studies [6].

We also note that reporting effects for men and women does not require new data collection. Gender is a variable that is always assessed in clinical trials. Hence, re-analysis of existing trials, either by stratifying results by gender or by analysing gender effects directly, would only require modest additional research investments. While existing studies may not be powered to detect differences between male and female patients, meta-analysis across trials is ideally suited to analyse gender differences, as long as authors report gender-stratified data.

Our systematic review of gender as a moderator of alcohol use disorder treatment response indicates we do not know whether clinical guidelines should be gender specific. The question remains, do men and women respond differently to evidence-based treatments for alcohol use disorder? We hope this commentary may spur the research needed to find the answer.

\section{Funding}

The work was funded by the Department of Defense Psychological Health Center of Excellence and the RAND Corporation. The findings and conclusions are those of the authors and do not necessarily represent the views of the Department of Defense or the RAND Corporation.

\section{References}

1. Grant BF, Goldstein RB, Saha TD, Chou SP, Jung J, et al. (2015) Epidemiology of DSM-5 Alcohol Use Disorder: Results from the National Epidemiologic Survey on Alcohol and Related Conditions III. JAMA Psychiatry 72: 757-766. [Crossref]

2. Ramchand R, Rudavsky R, Grant S, Tanielian T, Jaycox L (2015) Prevalence of, risk factors for, and consequences of posttraumatic stress disorder and other mental health problems in military populations deployed to Iraq and Afghanistan. Curr Psychiatry Rep 17: 37. [Crossref]

3. 2015 Health Related Behaviors Survey: Summary Findings and Policy Implications Available from: https://www.rand.org/pubs/research_briefs/RB9955.html

4. VA/DoD Clinical Practice Guideline for the Management of Substance Use Disorders. Available from: https://www.healthquality.va.gov/guidelines/MH/sud/ VADoDSUDCPGRevised22216.pdf 
5. Booth M, Rutter CM, Apaydin E, Karimi G, Shanman RM, et al. (2019) Gender Differences in Response to Alcohol Use Disorder Treatment: A Systematic Review. Santa Monica, CA: RAND Corporation, Newberry, Sydne. Available from: https:// www.rand.org/pubs/research_reports/RR2178.html.
6. Cohen MZ, Thompson CB, Yates B, Zimmerman L, Pullen CH (2015) Implementing common data elements across studies to advance research. Nurs Outlook 63: 181-188. [Crossref]

Copyright: (02019 Sydne N. This is an open-access article distributed under the terms of the Creative Commons Attribution License, which permits unrestricted use, distribution, and reproduction in any medium, provided the original author and source are credited. 